\title{
EVM's Java Dynamic Memory Manager and Garbage Collector
}

\author{
Sang-Yun Lee ${ }^{1}$ and Byung-Uk Choi ${ }^{2}$ \\ ${ }^{1}$ Dept. of Electronical Telecommunication Engineering, Hanyang University, Seoul, Korea \\ sylleedetri.re.kr \\ ${ }^{2}$ Division of Information and Communications, Hanyang University, Seoul, Korea \\ buchoi@hanyang.ac.kr
}

\begin{abstract}
In this paper, we propose the dynamic memory manager and garbage collector for the embedded Java virtual machine. In order to facilitate to memory allocation and deallocation fast, the memory manager divides a heap into various sizes and thus manages it with the unit of block which is a set of identical cell. And we propose a new 4-color based Mark \& Sweep garbage collector in order to support a multi-thread.
\end{abstract}

Keywords: Garbage Collector, Memory Management, Mark \& Sweep, EVM.

\section{Introduction}

We have developed the Embedded Java Virtual Machine (EVM), in which we introduce the dynamic memory manager and the garbage collector implemented in the EVM. The proposed memory manager divides the memory into a block and a cell. The cell is a constant sized-memory and thus becomes the unit of the object allocation and garbage collection. A block manages the cells with identical size through a linked-list. Dividing the memory into a block and a cell facilitate allocation of memory fast, because it quickly finds out the cell that fits the size of required object.

The proposed garbage collector is based on 3-color Mark \& Sweep collector [1]. This garbage collector operates well without any problem in a single thread. However, in the Java program operating in a multi-thread, there is a problem that the object is collected as garbage, when a garbage collector operates before the object allocated in the other thread is defined as a root set. In this paper, we propose the 4-color Mark \& Sweep garbage collector in order to solve this problem.

\section{Design of Dynamic Memory Manager and Garbage Collector}

We designed the memory structure considering the following criteria. Firstly, the memory allocation and deallocation speed must be fast. Secondly, we must consider that the programs operating in an embedded system normally operates with small sized objects. Thirdly, the large object must be handled with special care. Fourthly, the memory fragmentation must be small. Lastly, the expansion of memory capacity must be possible. 
We adopted a free-list [2] to satisfy them. Fig. 1 shows the memory block structure designed with the free-list.

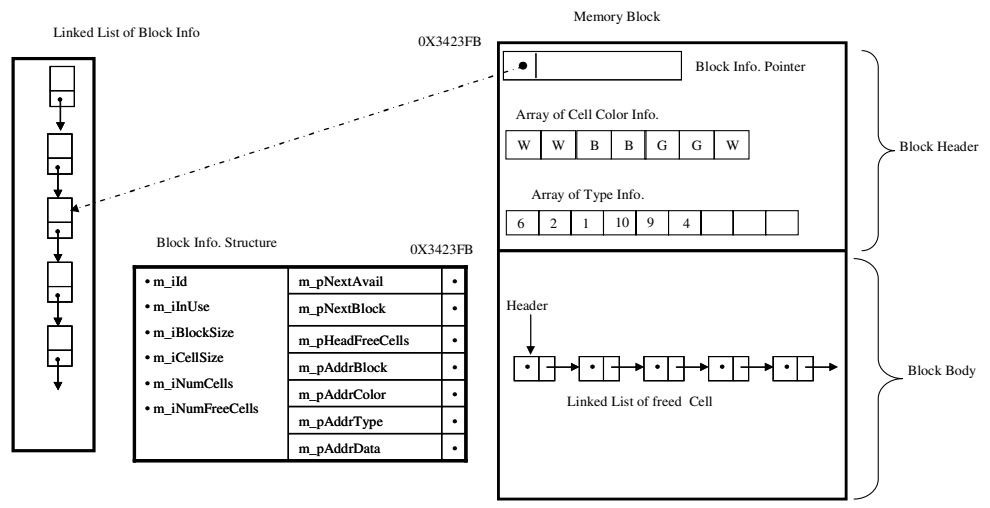

(a) Memory Region managed by the System (b) Memory Region managed by the VM

Fig. 1. The memory architecture

The memory area is divided into the system management memory region and the VM management memory region; the former obtains the whole heap from the system and stores the objects for controlling the latter and the latter allocates or deallocates an object dynamically in a virtual machine. And the latter is further divided into a block header and a block body. In the block header, the pointer of the block information structure, the color information of cells belonging to the block, and type information of memory allocation are stored.

The number of blocks is determined by the initial hip size assigned when the Java virtual machine is initiated. And the block memory is created during the Java virtual machine's initialization. The memory is additionally allocated from the system if the requested memory allocation exceeds the maximum heap size.

The Memory Map has three types. The AvailableBlock is the block with an available cell. The EmptyBlock is the place where all cells are deallocated by the garbage collector. FilledBlock is the block with no more available cells. Firstly, the available block is searched in the AvailableBlock. and the cell to be allocated is then appointed. If it is unable to find the cell in the AvailableBlock, new cell is allocated in the EmptyBlock. A new block and a new cell are created if it is unable to more allocate in the EmptyBlock. Specially, when there is request for allocation greater than 4,040 bytes it is allocated in the EmptyBlock.

The proposed garbage collector is based on the 3-color garbage collector that is easy to implement and its overhead is low in comparison with the other algorithm. The 3-color based Mark \& Sweep garbage collector runs normally in the single threaded environment, however it can cause serious problems in the multi-threaded environment $[3,4]$. 


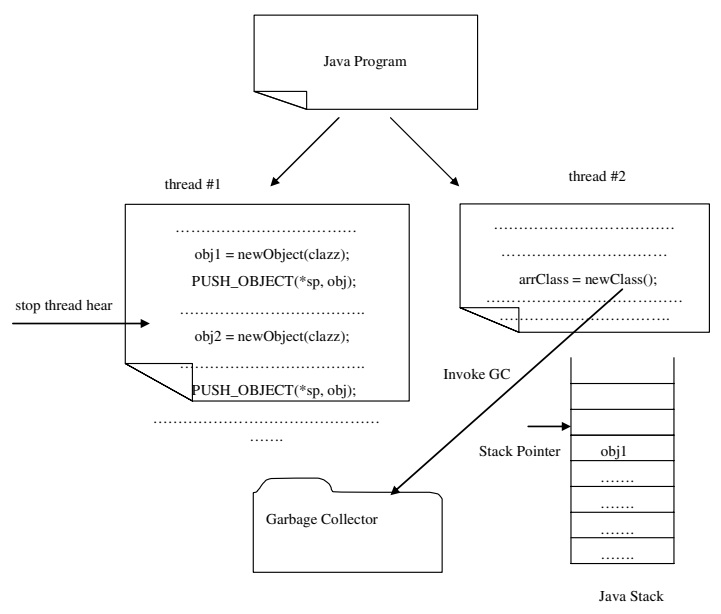

Fig. 2. The Problem of 3-color based Mark \& Sweep Garbage Collector

Fig. 2 is an example of programs in which the Java application runs two threads at the same time. The source code of the thread \#1 and thread \#2 is the internal implementation code of the Java virtual machine. In the thread \#1, the objects were stored within an obj1 and an obj2. The obj1 was stored in the Java stack by performing the PUSH OBJECT() function.

By the way, assume that the thread \#1 was stopped due to the thread \#2's calling the garbage collector, before an obj2 was stored in the Java stack. As shown in the Java stack memory structure, an obj 2 was allocated from the memory manager but it is in the state of not going over to the Java stack. Therefore, the garbage collector determines the obj 2 as garbage and collects it. Consequently, when the garbage collection is finished, thread \#1 runs again the obj2 cannot actually indicate the memory address value.

We propose the 4-color based Mark \& Sweep garbage collector to solve this problem. The proposed garbage collector has additional color more called YELLOW besides 3 colors used in 3-color based Mark \& Sweep garbage collector. The object with YELLOW color represents that it is not yet included in the root set but it is excluded from target object during the garbage collection. When an object is created, the object does not have the WHITE color but the YELLOW color. And it is changed to the WHITE color on appropriate time. We managed the memory for the YELLOW color object specially.

\section{Experimental Result}

We measured how the memory fragmentation changed as the number of object increased. We classified the objects into three classes for an experiment. In group A, we continuously created array of integer of which size is 10 . In group B, we continuously created array of double continuously of which size is 10 . In group $\mathrm{C}$, we continuously created array of integer, double, and float of which size is 10. Fig. 3 shows the experimental result. 


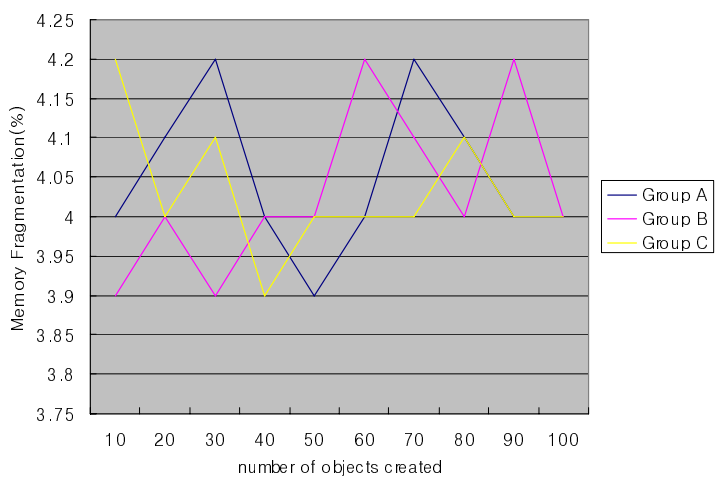

Fig. 3. Fragmentation variation according to creation of object

As shown in Fig. 3, we can know that although the number of objects created increases the memory fragmentation ratio is steadily maintained.

\section{Conclusion}

In this paper, we proposed the memory manager and garbage collector of the EVM, an embedded Java virtual machine. The memory manager adopted the freelist. This method has the disadvantage of the memory fragmentation but the memory allocation is fast. We designed the memory manage with blocks and cells efficiently. So, the internal and external memory fragmentation was very low (within 10\%).

We adopted 3-color based Mark \& Sweep garbage collector, whereas we proposed 4-color based Mark \& Sweep garbage collector to support a multi-threaded environment.

\section{References}

1. Narendran Sachindran, J. Eliot B. Moss, Emery D. Berger: MC $^{2}$ : High-Performance Garbage Collection for Memory-Constrained Environments, In proceedings of 19th annual ACM SIGPLAN conference on OOPSLA (Oct. 2004) 81-98

2. H. Toledano, M. T.: Toward an Analysis of Garbage Collection Techniques for Embedded Real-Time Java systems, 12th IEEE International Conference on Embedded and Real-Time Computing System and Applications (2006) 97-100

3. H. Inoue, D. Stefanovic, S. Forrest: On the prediction of Java object lifetimes, IEEE Transactions on Computers, Vol. 55. Issue 7 (July 2006) 880-892

4. W. Liu, Z. Chen, S. Tu: Research and analysis of garbage collection mechanism for realtime embedded java, In Proceedings of International Conference on Computer Supported Cooperative Work in Design (May 2004) 462-468 